\title{
Host range of 14 mycobacteriophages in Mycobacterium ulcerans and seven other mycobacteria including Mycobacterium tuberculosis - application for identification and susceptibility testing
}

\author{
Jan Rybniker, ${ }^{1}$ Stefanie Kramme ${ }^{1}$ and Pamela L. Small ${ }^{2}$ \\ ${ }^{1}$ Department of Microbiology, Bernhard Nocht Institute for Tropical Medicine, \\ Bernhard-Nocht-Str. 74, 20359 Hamburg, Germany \\ ${ }^{2}$ Department of Microbiology, University of Tennessee, Knoxville, TN 37996, USA
}

\begin{abstract}
The host range of well-characterized mycobacteriophages, such as D29 and TM4, has been determined, together with that of more recently isolated mycobacteriophages, in Mycobacterium ulcerans, Mycobacterium tuberculosis, Mycobacterium bovis BCG, Mycobacterium avium, Mycobacterium marinum, Mycobacterium scrofulaceum, Mycobacterium fortuitum and Mycobacterium chelonae. Here, a set of virulent phages for M. ulcerans, a pathogen with a dramatic increase of incidence over the last decade, is demonstrated. In this work, a mycobacteriophage replication assay was adapted for the identification and rifampicin-susceptibility testing of M. ulcerans. Mycobacteriophages have generated a number of useful tools and enabled insights into mycobacterial genetics. With regard to the neglected pathogen $M$. ulcerans, the findings presented in this work allow the application of a large range of phage-based vectors and markers. The potential of phage therapy can now be evaluated for this extracellular pathogen.
\end{abstract}

Accepted 20 September 2005

\section{INTRODUCTION}

Mycobacteriophages have proved to be useful genetic tools for the manipulation of pathogenic mycobacteria. The construction of conditionally replicating mycobacteriophages based on phage TM4 and D29 has enabled knock-out mutants to be generated in many mycobacterial species (Bardarov et al., 1997; Harris et al., 1999; Rybniker et al., 2003). Furthermore, mycobacteriophages are used as diagnostic markers to improve and expedite the recognition of pathogenic mycobacteria in patient samples, as well as the drug resistance of these pathogens. Commercial kits based on phage technology are now available (Alcaide et al., 2003; Muzaffar et al., 2002). In addition, TM4 and D29 luciferase reporter phages have been constructed for the same purpose (Jacobs et al., 1993; Pearson et al., 1996). Broxmeyer et al. (2002) demonstrated that mycobacteriophages have potential as antimycobacterial agents for infections caused by Mycobacterium avium and Mycobacterium tuberculosis when using a non-pathogenic mycobacterium as a delivery system to intracellularly located bacteria. Over 250 mycobacteriophages have been described, and typing schemes have been developed for many mycobacterial species (Crawford \& Bates, 1984).

Abbreviation: FAS, ferrous ammonium sulfate.
For the successful application of phages, the knowledge of their host range is a prerequisite. However, data on the host range of the recently isolated mycobacteriophages are rare and have often been communicated orally. This led us to examine 14 mycobacteriophages for their ability to infect and replicate in several fast- or slow-growing mycobacteria, including M. tuberculosis, Mycobacterium ulcerans and $M$. avium. The complete nucleotide sequences of eight of the phages used in this study are available through GenBank (Pedulla et al., 2003). The determination of the host-range for M. ulcerans may help to improve our understanding of the pathogenesis of this organism, as well as the diagnosis of this neglected pathogen. The slow-growing M. ulcerans causes Buruli ulcer, a serious ulcerating skin disease common in many tropical countries (Thangaraj et al., 2003). This major mycobacteriosis represents the third most common mycobacterial infection in immunocompetent patients (Weir, 2002). To date, the accepted treatment is surgical resection of infected skin followed by grafting. However, efforts are being made to evaluate the potential of drug treatment in larger clinical trials (Espey et al., 2002). It is likely that future treatment schemes will be based on the antituberculotic drug rifampicin. However, rifampicinresistant $M$. ulcerans mutants have already been isolated from experimental mouse lesions after monotherapy with this drug (Marsollier et al., 2003). This led us to adapt phage-based rifampicin-susceptibility tests to M. ulcerans. 
As with other mycobacterial diseases, there are no routine serological tests available for Buruli ulcer. Primary cultures for the diagnosis of $M$. ulcerans may take 6-8 weeks for a positive result. There is a need for affordable alternative diagnostic methods. Phages used for the rapid identification of a given mycobacterium from patient samples lack specificity. They can be useful if they show a superior sensitivity to an examination by acid-fast smear microscopy in a setting in which PCR detection is not available or not affordable. Investigations of the phage-based detection of M. tuberculosis from sputum samples have given contradictory results (Alcaide et al., 2003; McNerney et al., 2004; Muzaffar et al., 2002). In contrast to staining and PCR methods, phage assays can detect viable mycobacteria only, which is the principle of susceptibility tests. Here, we show that specificity for the detection of $M$. ulcerans can be enhanced by splitting the sample and pre-incubating aliquots at $41{ }^{\circ} \mathrm{C}$ and $32^{\circ} \mathrm{C}$ for $24 \mathrm{~h}$. M. ulcerans cells incubated at the higher temperature do not allow phage replication and detection, whereas a mycobacterium with a different optimal growth temperature, such as $M$. tuberculosis, will give a positive result at both temperatures.

\section{METHODS}

Bacterial strains, mycobacteriophages, media and culture methods. The mycobacterial strains used in this work are listed in Table 1. All strains were grown in Middlebrook 7H9 broth (Difco) enriched with $10 \%$ OADC (oleic acid, BSA, dextrose and catalase) (Difco) in $175 \mathrm{~cm}^{2}$ tissue-culture flasks (Falcon) at $37^{\circ} \mathrm{C}$, except for M. marinum and M. ulcerans, which were grown at $32^{\circ} \mathrm{C}$.
Rifampicin-resistant $M$. ulcerans isolates were generated by plating $5 \times 10^{10}$ c.f.u. of a clinical isolate from Ghana on Middlebrook 7H10 plates containing $10 \mu \mathrm{g}$ rifampicin $\mathrm{ml}^{-1}$ (Sigma).

Except for phage TM4, the primary isolation of all mycobacteriophages used in this study employed Mycobacterium smegmatis as host. Mycobacteriophage D29 was isolated from soil by Froman et al. (1954). Doke et al. (1960) isolated the temperate phage L5 from cultures of $M$. smegmatis. TM4 was isolated from an M. avium culture after treatment with mitomycin C (Timme \& Brennan, 1984). Bxz2 was isolated from environmental samples from the Bronx Zoo by the William R. Jacobs laboratory at the Albert Einstein College of Medicine, New York. The remaining phages were isolated in Graham F. Hatfull's laboratory at the University of Pittsburgh, except for phage Che8, which was isolated in Chennai, India. The phages are listed in Table 2.

Growth of mycobacteriophage. All mycobacteriophages were propagated in M. smegmatis $\mathrm{mc}^{2} 155$, as described elsewhere (Sarkis \& Hatfull, 1998). In brief, $250 \mu \mathrm{l}$ of cells grown to an $\mathrm{OD}_{600}$ of $1 \cdot 0$ was combined with approximately $10^{5}$ phage particles and $3 \mathrm{ml}$ molten top agar $(4 \cdot 7 \mathrm{~g}$ Middlebrook $7 \mathrm{H} 9$ broth base, $7 \mathrm{~g}$ Bacto agar (Difco) in $900 \mathrm{ml}$ distilled water enriched with $25 \mathrm{ml} 40 \%$ glucose and $10 \mathrm{ml} 0 \cdot 1 \mathrm{M} \mathrm{CaCl}_{2}$ ). This mixture was poured on $7 \mathrm{H} 10$ agar plates containing $10 \% \mathrm{OADC}$ and incubated overnight at $37^{\circ} \mathrm{C}$. The plates were covered with $5 \mathrm{ml}$ phage buffer $(10 \mathrm{mM}$ Tris, $\mathrm{pH} 7 \cdot 5,1 \mathrm{mM} \mathrm{MgSO}$, $70 \mathrm{mM} \mathrm{NaCl}$ ) for $4 \mathrm{~h}$ at $4{ }^{\circ} \mathrm{C}$, and phage particles were harvested by transferring the buffer into a sterile container. After passing the phage suspension through a $0.2 \mu \mathrm{m}$ pore-size filter, the phage titre was determined using a standard spot test. Using this method, titres $>10^{10}$ p.f.u. $\mathrm{ml}^{-1}$ were reached for all phages.

Host-range determination. Mycobacteria were grown to an $\mathrm{OD}_{600}$ of $1 \cdot 0$. If necessary, clumps were dispersed by passing the bacterial suspension several times through a 25 -gauge needle. A volume of $500 \mu \mathrm{l}$ was added to $3 \mathrm{ml}$ top agar containing $1 \mathrm{mM}$

Table 1. Mycobacterial strains used in this study

\begin{tabular}{|c|c|c|}
\hline Species & Strain & Reference or source \\
\hline M. tuberculosis & $\mathrm{H} 37 \mathrm{Rv}$ & ATCC $^{*}$ \\
\hline M. tuberculosis complex, $n=5$ & Clinical isolates & $\mathrm{BNI} \dagger$ \\
\hline Mycobacterium bovis & BCG Pasteur & ATCC \\
\hline M. avium & $3746 / 02$ & Fz-Borstel $\ddagger$ \\
\hline M. avium & 701 & P. L. Small, UTK\$ \\
\hline M. avium & 702 & P. L. Small, UTK \\
\hline M. scrofulaceum & 1320 & P. L. Small, UTK \\
\hline M. scrofulaceum & 1315 & P. L. Small, UTK \\
\hline M. ulcerans & 1615 (ATCC 35840) & Isolated in Malaysia, ATCC \\
\hline M. ulcerans & 1615 mycolactone $^{-}$mutant & P. L. Small, UTK; Stinear et al. (2004) \\
\hline M. ulcerans, $n=15$ & Clinical isolates & All endemic continents, BNI \\
\hline M. ulcerans & RifR (rifampicin-resistant) & BNI (this work) \\
\hline M. marinum & ATCC 927 & ATCC \\
\hline M. marinum & 565 & P. L. Small, UTK \\
\hline M. fortuitum & 1529 & P. L. Small, UTK \\
\hline M. chelonae & 1543 & P. L. Small, UTK \\
\hline M. smegmatis & $\mathrm{mc}^{2} 155$ & BNI; Snapper et al. (1990) \\
\hline
\end{tabular}

${ }^{\star}$ American Type Culture Collection.

$\nmid$ Bernhard Nocht Institute for Tropical Medicine.

$\ddagger$ National Reference Center for Mycobacteria, Forschungszentrum Borstel, Germany.

$\S$ Dr P. L. Small, University of Tennessee, Department of Microbiology. 
Table 2. Host range of 14 mycobacteriophages in eight mycobacterial species

1, M. smegmatis $\mathrm{mc}^{2} 155$; 2, M. tuberculosis H37Rv; 3, M. tuberculosis 371; 4, BCG Pasteur; 5, M. avium 701; 6, M. avium 702; 7, M. avium $3746 / 02 ; 8$, M. scrofulaceum 1320; 9, M. scrofulaceum 1315; 10, M. ulcerans 1615; 11, M. ulcerans 1615M; 12, M. ulcerans M18 (Ghana); 13, M. ulcerans S12 (Ghana); 14, M. marinum ATCC 927; 15, M. marinum 565; 16, M. fortuitum 1529; 17, M. chelonae 1543. P, Plaque formation.

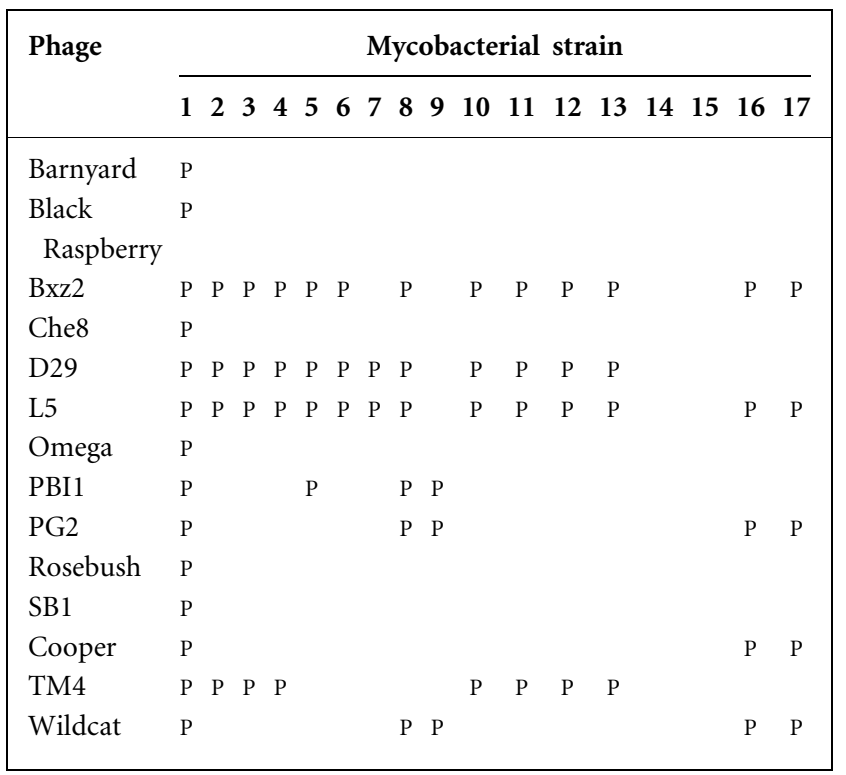

$\mathrm{CaCl}_{2}$ and poured onto $7 \mathrm{H} 10$ agar plates enriched with $1 \mathrm{mM} \mathrm{CaCl}$ and OADC. For each phage, a serial dilution was prepared in phage buffer. Twenty-microlitre volumes of the original stock and of each dilution were pipetted onto the bacterial lawn, and the spots were allowed to dry completely. Plates were incubated for 4 days for the fast-growing mycobacteria, and for up to 6 weeks for the slowgrowing strains, at the optimal temperature for the individual strain. All experiments were performed in triplicate.

Phage amplification assay. Fifteen rifampicin-sensitive $M$. ulcerans clinical isolates (confirmed by standard agar dilution tests) from the major endemic regions and one rifampicin-resistant isolate were grown in Middlebrook 7H9 broth enriched with $10 \%$ OADC. Five $1.5 \mathrm{ml}$ screw-capped microcentrifuge tubes were loaded with $1 \mathrm{ml}$ of each culture and centrifuged at 5000 r.p.m. The supernatant was discarded and the pellet resuspended in $1 \mathrm{ml}$ of fresh Middlebrook 7H9 broth supplemented with $10 \%$ OADC. Of the five tubes, one was incubated at $41{ }^{\circ} \mathrm{C}$ and the second at $32{ }^{\circ} \mathrm{C}$ for $24 \mathrm{~h}$. In the three remaining tubes, rifampicin was added at 1, 5 and $10 \mu \mathrm{g} \mathrm{ml}^{-1}$, and incubation took place at $33^{\circ} \mathrm{C}$ for $24 \mathrm{~h}$. From each sample, $50 \mu \mathrm{l}$ was transferred to new $1.5 \mathrm{ml}$ tubes, and $50 \mu \mathrm{l}$ aliquots of phage D29 at $10^{7}$ p.f.u. $\mathrm{ml}^{-1}$ were added. The D29 phage stock had been diluted beforehand in $7 \mathrm{H} 9$ broth with $10 \%$ OADC and $2 \mathrm{mM} \mathrm{CaCl}_{2}$. Phage infection was allowed to take place for $90 \mathrm{~min}$; temperatures were the same as in the prior $24 \mathrm{~h}$ incubation period. Inactivation of exogenous phage particles was achieved by the addition of $2 \mathrm{mM}$ ferrous ammonium sulfate (FAS, Carl Roth) (McNerney et al., 1998). After vigorous vortexing and incubation of the samples for $5 \mathrm{~min}$ at room temperature, $20 \mu \mathrm{l}$ of each aliquot was spotted on $M$. smegmatis $\mathrm{mc}^{2} 155$ top agar indicator plates. The presence of plaques after an overnight incubation at $33^{\circ} \mathrm{C}$ was taken to indicate a $M$. ulcerans-positive or rifampicin-resistant isolate. The test was performed in the same manner with five clinical isolates of the M. tuberculosis complex.

Electron microscopy. A CsCl-purified stock of mycobacteriophage D29 was prepared as described previously (Sarkis \& Hatfull, 1998). D29 ( $10^{9}$ p.f.u.) was incubated for $30 \mathrm{~min}$ with $10^{7}$ c.f.u. of $M$. ulcerans 1615 at $37^{\circ} \mathrm{C}$. A $5 \mu \mathrm{l}$ drop of the sample was placed on a freshly glow-discharged, collodion and carbon-coated grid. After $1 \mathrm{~min}$, the grid was washed with $10 \mathrm{mM}$ ammonium acetate, and then stained for $30 \mathrm{~s}$ with $0 \cdot 75 \%$ uranyl formate. Samples were examined and photographed with a Hitachi H800 scanning transmission electron microscope operating at $100 \mathrm{kV}$.

\section{RESULTS AND DISCUSSION}

The results for the plaque formation on the tested mycobacterial species are given in Table 2. For each mycobacterium tested, only the observation of individual plaques at high dilutions of the phage solution was used as a proof for growth support. Plaque formation that takes place only at the lowest dilutions is often due to 'killing from without'. This phenomenon occurs when a very high phage : cell ratio leads to lysis of the bacteria without productive phage infection (Sarkis \& Hatfull, 1998).

Six mycobacteriophages did not form plaques on any species other than M. smegmatis, the mycobacterium which was used for their primary isolation from environmental samples. A cluster of four phages, namely D29, TM4, L5 and Bxz2, seemed to have the broadest host range, with at least three of these phages forming plaques on all of the slow-growing species, except for M. marinum and one strain of M. scrofulaceum. The genomes of these four phages have detectable sequence similarity, which might be one reason for their shared host range (Pedulla et al., 2003). $\mathrm{CaCl}_{2}$ is important for the infection of a number of mycobacteriophages. In our experiments, the $\mathrm{CaCl}_{2}$ concentration was maintained at $1 \mathrm{mM}$. It is possible that different concentrations alter the host range of some phages.

\section{Host range in $M$. ulcerans and $M$. marinum}

The host-range spectrum obtained for $M$. ulcerans was similar to that of M. tuberculosis and Mycobacterium bovis BCG. This opens the possibility to apply phage-based diagnostic tools, as well as vectors for improved transformation, to this important pathogen. D29 seems to be a good choice for mycobacteriophage assays for the detection of M. ulcerans in patient samples or to investigate the drug resistance of mycobacterial isolates. This phage proved to be lytic in $M$. ulcerans species which originated from four different continents. M. ulcerans shows genetic heterogeneity and variable phenotypic characteristics among strains of different geographic origins (Portaels et al., 1996). Even so, the lytic phenotype of D29 is stable in M. ulcerans, as seems to be the case for most strains of M. tuberculosis (Banaiee et al., 2003; McNerney et al., 2004).

Additionally, phage therapy could be considered for $M$. ulcerans infections. In Buruli ulcer, mycobacteria are 
typically found extracellularly, where they might be immediately accessible by lytic phages. Again, phage D29 might be the best candidate for this purpose, since the plaques are relatively large and adsorption of phage particles seems to be efficient (Fig. 1).

M. ulcerans produces the polyketide-derived macrolide mycolactone, which is required for the tissue damage that characterizes Buruli ulcer (George et al., 1999; Mve-Obiang et al., 2003). Although mycolactone is a major component of the cell surface of M. ulcerans, it does not seem to be involved in the binding of phages or the injection of phage DNA, since the avirulent mycolactone-negative mutant $1615 \mathrm{M}$ showed the same host range as the wild-type strain.

Interestingly, none of the tested phages formed plaques on two strains of M. marinum. In the case of TM4 and D29 at least, this was most likely due to an intracellular inhibition of phage replication, rather than a receptor-receptor binding protein mismatch, since shuttle phasmids based on these two phages are able to transfect M. marinum (Rybniker et al., 2003). This restriction of phage growth in M. marinum might be of advantage to phage-derived diagnostic tools for the rapid identification of $M$. ulcerans. The observation of plaque formation in M. ulcerans, but not in M. marinum, allows the discrimination of these two pathogens which share the ability to cause dermal diseases and have a common optimal growth temperature of $32^{\circ} \mathrm{C}$. However, additional M. marinum strains need to be tested to confirm this phenotype.

\section{Host range in mycobacteria other than M. ulcerans and M. marinum}

Except for Bxz2, none of the recently isolated phages formed plaques on $M$. tuberculosis or M. bovis BCG, showing that phages isolated in the fast-growing M. smegmatis are often

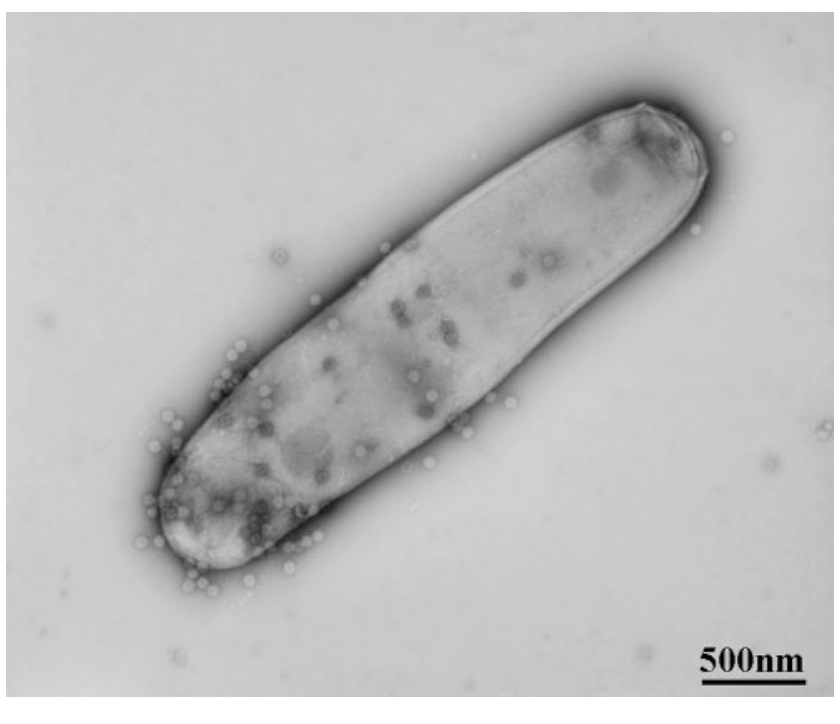

Fig. 1. Phage D29 adsorbed to M. ulcerans 1615. confined to this mycobacterium or other fast-growing species. It is likely that environmental samples, for example from compost, may contain additional phages, and the use of slow-growing species such as M. bovis BCG should be considered when searching for wild-type phages with a broader or different host spectrum.

Interestingly, our three M. avium strains, as well as strains of the closely related M. scrofulaceum, were resistant to TM4, and this differs from observations made by others (FoleyThomas et al., 1995). Since TM4 was induced from $M$. avium, it is possible that the restriction of replication observed in this mycobacterium is due to a lysogenic state of TM4 which confers superinfection immunity. Also, $M$. avium and $M$. scrofulaceum showed differing host ranges at the subspecies level, which was not observed in the other mycobacterial species tested. For example, Bxz2 formed plaques on M. avium 701 and 702, but not on M. avium 3746/02. Also, phage D29 formed plaques on M. scrofulaceum 1320 , but not on $M$. scrofulaceum 1315 . This failure to form plaques on all isolates of one species impedes the use of mycobacteriophage detection assays for the M. avium-M. scrofulaceum complex, and the possibility of such a phenotype should be kept in mind when using tools based on this phage. It has been stated elsewhere that phage D29 shows a very broad host range, infecting a wide variety of slowgrowing mycobacteria, with the exception of $M$. avium (McNerney et al., 2004). Again, this differs from our observations. D29 readily infected all three M. avium isolates tested. Hatfull \& Sarkis (1993) describe the ability of phage L5 to infect some substrains of M. avium. It is likely that phage D29 shows a similar phenotype; the fact that our $M$. avium strains allowed L5 and D29 growth supports this hypothesis.

However, in the fast-growing strains, a remarkable observation was plaque formation of phage L 5 but not of D29 in the M. fortuitum-chelonae complex. These two phages are very closely related, with extensive detectable DNA sequence similarity (Ford et al., 1998; Pedulla et al., 2003). Also, D29 is a homo-immune relative to L5.

\section{Phage amplification assay for detection of rifampicin-resistant $M$. ulcerans and discrimination between $M$. ulcerans and M. tuberculosis}

Several protocols for phage-based susceptibility tests and identification of $M$. tuberculosis from patient samples have been published (Albert et al., 2002; Butt et al., 2004; Simboli et al., 2005). These protocols were successfully adapted to $M$. ulcerans. The fact that the growth of M. ulcerans as well as the phage replication in this host is strongly restricted to temperatures below $35^{\circ} \mathrm{C}$ can be used for differentiation between M. ulcerans and M. tuberculosis, both of which cause dermal disease. Plaque formation on indicator plates after a $24 \mathrm{~h}$ incubation period at both $33^{\circ} \mathrm{C}$ and $41{ }^{\circ} \mathrm{C}$ indicates a M. ulcerans-negative sample, whereas plaque formation on the $33^{\circ} \mathrm{C}$ sample only will be M. ulcerans positive (Table 3, 
Table 3. Phage amplification test

Incubation time prior to the plaque test was $24 \mathrm{~h}$. P, Plaque formation; (P), few plaques; NT, not tested. See also legend of Fig. 2.

\begin{tabular}{|c|c|c|c|c|c|}
\hline Strain & $\begin{array}{l}\text { Incubation } \\
\text { at } 32^{\circ} \mathrm{C}\end{array}$ & $\begin{array}{c}\text { Incubation } \\
\text { at } 41^{\circ} \mathrm{C}\end{array}$ & $\begin{array}{c}1 \mu \mathrm{g} \\
\text { rifampicin } \mathrm{ml}^{-1}\end{array}$ & $\begin{array}{c}5 \mu \mathrm{g} \\
\text { rifampicin } \mathrm{ml}^{-1}\end{array}$ & $\begin{array}{l}10 \mu \mathrm{g} \\
\text { rifampicin } \mathrm{ml}^{-1}\end{array}$ \\
\hline M. ulcerans, $n=15$ & $\mathrm{P}$ & & (P) & & \\
\hline M. tuberculosis, $n=5$ & $\mathrm{P}$ & $\mathrm{P}$ & NT & NT & NT \\
\hline
\end{tabular}

Fig. 2). In two M. ulcerans isolates, some plaques were observed after the $24 \mathrm{~h}$ incubation period at $41^{\circ} \mathrm{C}$. Here, increasing the incubation time to $48 \mathrm{~h}$ improved the performance of the test, with no plaques visible on the indicator plate. Also the rifampicin-susceptibility test correlated well with results from rifampicin agar-dilution tests. In the rifampicin-sensitive isolates, plaque formation was decreased in a concentration-dependent manner: no plaques were visible at $10 \mu \mathrm{g}$ rifampicin $\mathrm{ml}^{-1}$. The rifampicinresistant isolate $M$. ulcerans RifR showed plaque formation at all three concentrations. We found the performance of the phage amplification test to be easy and fast, giving positive results within $48 \mathrm{~h}$. The test performed equally well when using M. ulcerans colonies picked from Loewenstein-Jensen agar (data not shown). This test might be an alternative to

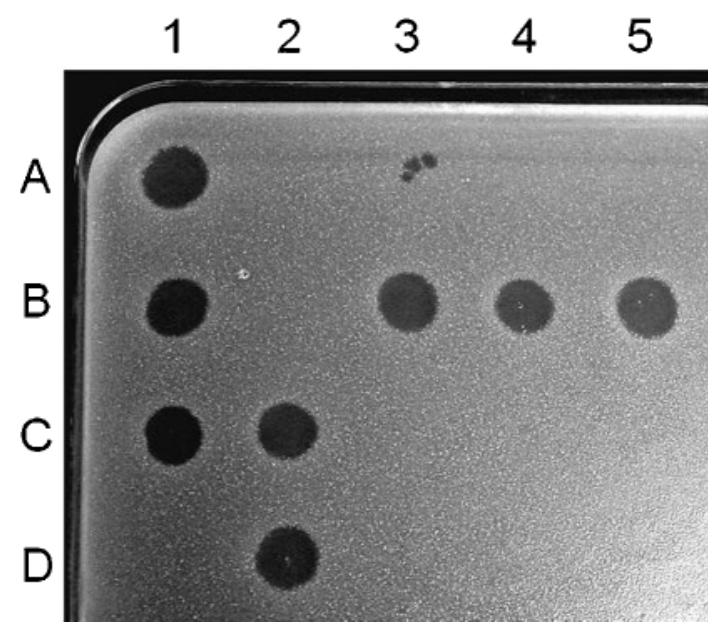

Fig. 2. Phage-based susceptibility and identification test. Before the addition of phage D29, M. ulcerans and M. tuberculosis strains were pre-incubated as indicated below. The plaque pattern allowed discrimination between rifampicin-resistant and -sensitive isolates (lines A3-A5 and lines B3-B5), and between $M$. ulcerans and $M$. tuberculosis (lines A1-A2, B1-B2, C1-C2). Line $A$, rifampicin-sensitive $M$. ulcerans isolate. $A 1,24 \mathrm{~h}$ preincubation at $32{ }^{\circ} \mathrm{C} ; \mathrm{A} 2,24 \mathrm{~h}$ pre-incubation at $41^{\circ} \mathrm{C} ; \mathrm{A} 3,24 \mathrm{~h}$ pre-incubation with $1 \mu \mathrm{g}$ rifampicin $\mathrm{ml}^{-1} ; \mathrm{A} 4,5 \mu \mathrm{g}$ rifampicin $\mathrm{ml}^{-1} ; \mathrm{A} 5,10 \mu \mathrm{g}$ rifampicin $\mathrm{ml}^{-1}$. Line $\mathrm{B}$, rifampicin-resistant M. ulcerans RifR, pre-incubation of B1-B5 like A1-A5. Line $\mathrm{C}$, M. tuberculosis complex, pre-incubation of $\mathrm{C} 1-\mathrm{C} 2$ like $\mathrm{A} 1-\mathrm{A} 2$. D1, phage only with 2 mM FAS; D2, phage only. expensive diagnostic tools that are dependent on the supply and storage of complex reagents. Particularly in acid-fastpositive patient samples, the rapid temperature-sensitivephage amplification test may differentiate between $M$. ulcerans and other mycobacteria, having major consequences concerning the management of the disease. A range of slow-growing mycobacterial species should be tested before the test is evaluated using samples such as swabs or skin biopsies. A laboratory performing phage-based tests could also improve its diagnostic performance with respect to tuberculosis, which is usually of high incidence in Buruli ulcer endemic regions.

In summary, we have shown that the mycobacteriophages D29, Bxz2, L5 and TM4 have a broad, and to some extent similar, host range among the slow-growing mycobacteria. With Bxz2, we present an additional virulent phage for $M$. tuberculosis. The data obtained for M. ulcerans open new possibilities for the diagnosis and genetic manipulation of this pathogen, whereas M. marinum seems to be broadly resistant to phage replication. To our knowledge, this has been the first systematic investigation of the phage host range in these two organisms.

\section{ACKNOWLEDGEMENTS}

We are grateful to Dr Graham F. Hatfull for the kind donation of the phages used in this work. Dr Gisela Bretzel provided some of the $M$. ulcerans strains. We thank Brian Ranger for the critical reading of the manuscript. This work was supported by NIH and a Koeln Fortune fellowship.

\section{REFERENCES}

Albert, H., Muzaffar, R., Mole, R. J. \& Trollip, A. P. (2002). Use of the FASTPlaque test for TB diagnosis in low-income countries. Int J Tuberc Lung Dis 6, 1130-1131 (author reply 1131-1132).

Alcaide, F., Gali, N., Dominguez, J., Berlanga, P., Blanco, S., Orus, P. \& Martin, R. (2003). Usefulness of a new mycobacteriophage-based technique for rapid diagnosis of pulmonary tuberculosis. J Clin Microbiol 41, 2867-2871.

Banaiee, N., Bobadilla-del-Valle, M., Riska, P. F., Bardarov, S., Jr, Small, P. M., Ponce-de-Leon, A., Jacobs, W. R., Jr, Hatfull, G. F. \& Sifuentes-Osornio, J. (2003). Rapid identification and susceptibility testing of Mycobacterium tuberculosis from MGIT cultures with luciferase reporter mycobacteriophages. J Med Microbiol 52, 557-561.

Bardarov, S., Kriakov, J., Carriere, C., Yu, S., Vaamonde, C., McAdam, R. A., Bloom, B. R., Hatfull, G. F. \& Jacobs, W. R., Jr 
(1997). Conditionally replicating mycobacteriophages: a system for transposon delivery to Mycobacterium tuberculosis. Proc Natl Acad Sci U S A 94, 10961-10966.

Broxmeyer, L., Sosnowska, D., Miltner, E., Chacon, O., Wagner, D., McGarvey, J., Barletta, R. G. \& Bermudez, L. E. (2002). Killing of Mycobacterium avium and Mycobacterium tuberculosis by a mycobacteriophage delivered by a nonvirulent mycobacterium: a model for phage therapy of intracellular bacterial pathogens. J Infect Dis 186, 1155-1160.

Butt, T., Ahmad, R. N., Afzal, R. K., Mahmood, A. \& Anwar, M. (2004). Rapid detection of rifampicin susceptibility of Mycobacterium tuberculosis in sputum specimens by mycobacteriophage assay. J Pak Med Assoc 54, 379-382.

Crawford, J. \& Bates, J. H. (1984). Phage typing of mycobacteria. In The Biology of Mycobacteria: a Sourcebook, pp. 309-351. Edited by G. P. Kubica \& L. G. Wayne. London: Academic Press.

Doke, S. (1960). Studies on mycobacteriohages and lysogenic mycobacteria. J Kumamoto Med Soc 34, 1360-1373.

Espey, D. K., Meyers, W. M., Portaels, F. \& 9 other authors (2002). A pilot study of treatment of Buruli ulcer with rifampin and dapsone. Int $J$ Infect Dis 6, 60-65.

Foley-Thomas, E. M., Whipple, D. L., Bermudez, L. E. \& Barletta, R. G. (1995). Phage infection, transfection and transformation of Mycobacterium avium complex and Mycobacterium paratuberculosis. Microbiology 141, 1173-1181.

Ford, M. E., Sarkis, G. J., Belanger, A. E., Hendrix, R. W. \& Hatfull, G. F. (1998). Genome structure of mycobacteriophage D29: implications for phage evolution. J Mol Biol 279, 143-164.

Froman, S., Will, D. W. \& Bogen, E. (1954). Bacteriophage active against virulent Mycobacterium tuberculosis. I. Isolation and activity. Am J Public Health 44, 1326-1333.

George, K. M., Chatterjee, D., Gunawardana, G., Welty, D., Hayman, J., Lee, R. \& Small, P. L. (1999). Mycolactone: a polyketide toxin from Mycobacterium ulcerans required for virulence. Science 283, 854-857.

Harris, N. B., Feng, Z., Liu, X., Cirillo, S. L., Cirillo, J. D. \& Barletta, R. G. (1999). Development of a transposon mutagenesis system for Mycobacterium avium subsp. paratuberculosis. FEMS Microbiol Lett 175, 21-26.

Hatfull, G. F. \& Sarkis, G. J. (1993). DNA sequence, structure and gene expression of mycobacteriophage L5: a phage system for mycobacterial genetics. Mol Microbiol 7, 395-405.

Jacobs, W. R., Jr, Barletta, R. G., Udani, R. \& 7 other authors (1993). Rapid assessment of drug susceptibilities of Mycobacterium tuberculosis by means of luciferase reporter phages. Science 260, 819-822.

Marsollier, L., Honore, N., Legras, P., Manceau, A. L., Kouakou, H., Carbonnelle, B. \& Cole, S. T. (2003). Isolation of three Mycobacterium ulcerans strains resistant to rifampin after experimental chemotherapy of mice. Antimicrob Agents Chemother 47, $1228-1232$.

McNerney, R., Wilson, S. M., Sidhu, A. M., Harley, V. S., al Suwaidi, Z., Nye, P. M., Parish, T. \& Stoker, N. G. (1998). Inactivation of mycobacteriophage D29 using ferrous ammonium sulphate as a tool for the detection of viable Mycobacterium smegmatis and $M$. tuberculosis. Res Microbiol 149, 487-495.

McNerney, R., Kambashi, B. S., Kinkese, J., Tembwe, R. \& GodfreyFaussett, P. (2004). Development of a bacteriophage phage replication assay for diagnosis of pulmonary tuberculosis. J Clin Microbiol 42, 2115-2120.

Muzaffar, R., Batool, S., Aziz, F., Naqvi, A. \& Rizvi, A. (2002). Evaluation of the FASTPlaqueTB assay for direct detection of Mycobacterium tuberculosis in sputum specimens. Int J Tuberc Lung Dis 6, 635-640.

Mve-Obiang, A., Lee, R. E., Portaels, F. \& Small, P. L. (2003). Heterogeneity of mycolactones produced by clinical isolates of Mycobacterium ulcerans: implications for virulence. Infect Immun 71, 774-783.

Pearson, R. E., Jurgensen, S., Sarkis, G. J., Hatfull, G. F. \& Jacobs, W. R., Jr (1996). Construction of D29 shuttle phasmids and luciferase reporter phages for detection of mycobacteria. Gene 183, 129-136.

Pedulla, M. L., Ford, M. E., Houtz, J. M. \& 17 other authors (2003). Origins of highly mosaic mycobacteriophage genomes. Cell 113, 171-182.

Portaels, F., Fonteyene, P. A., de Beenhouwer, H., de Rijk, P., Guedenon, A., Hayman, J. \& Meyers, M. W. (1996). Variability in $3^{\prime}$ end of 16S rRNA sequence of Mycobacterium ulcerans is related to geographic origin of isolates. J Clin Microbiol 34, 962-965.

Rybniker, J., Wolke, M., Haefs, C. \& Plum, G. (2003). Transposition of Tn5367 in Mycobacterium marinum, using a conditionally recombinant mycobacteriophage. J Bacteriol 185, 1745-1748.

Sarkis, J. S. \& Hatfull, G. F. (1998). Mycobacteriophages. In Mycobacteria Protocols, pp. 145-173. Edited by T. Parish \& N. G. Stoker. Totowa, NJ: Humana Press.

Simboli, N., Takiff, H., McNerney, R., Lopez, B., Martin, A., Palomino, J. C., Barrera, L. \& Ritacco, V. (2005). In-house phage amplification assay is a sound alternative for detecting rifampin-resistant Mycobacterium tuberculosis in low-resource settings. Antimicrob Agents Chemother 49, 425-427.

Snapper, S. B., Melton, R. E., Mustafa, S., Kieser, T. \& Jacobs, W. R., Jr (1990). Isolation and characterization of efficient plasmid transformation mutants of Mycobacterium smegmatis. Mol Microbiol 4, 1911-1919.

Stinear, T. P., Mve-Obiang, A., Small, P. L. \& 12 other authors (2004). Giant plasmid-encoded polyketide synthases produce the macrolide toxin of Mycobacterium ulcerans. Proc Natl Acad Sci U S A 101, 1345-1349.

Thangaraj, H. S., Phillips, R. O., Evans, M. R. \& Wansbrough-Jones, M. H. (2003). Emerging aspects of Buruli ulcer. Expert Rev Anti Infect Ther 1, 217-222.

Timme, T. L. \& Brennan, P. J. (1984). Induction of bacteriophage from members of the Mycobacterium avium, Mycobacterium intracellulare, Mycobacterium scrofulaceum serocomplex. J Gen Microbiol 130, 2059-2066.

Weir, E. (2002). Buruli ulcer: the third most common mycobacterial infection. Can Med Assoc J 166, 1691. 